\title{
The Beckenbach-Dresher inequality in the $\Psi$ - direct sums of spaces and related results
}

\author{
Ludmila Nikolova', Lars-Erik Persson ${ }^{2}$ and Sanja Varošanec ${ }^{3^{*}}$
}

\author{
* Correspondence: varosans@math. \\ hr \\ ${ }^{3}$ Department of Mathematics, \\ University of Zagreb, Zagreb, \\ Croatia \\ Full list of author information is \\ available at the end of the article
}

\begin{abstract}
Let $\tilde{\psi}:[0,1] \rightarrow \mathbf{R}$ be a concave function with $\tilde{\psi}(0)=\tilde{\psi}(1)=1$. There is a corresponding map $\|\cdot\|_{\tilde{\psi}}$ for which the inverse Minkowski inequality holds. Several properties of that map are obtained. Also, we consider the Beckenbach-Dresher type inequality connected with $\psi$-direct sums of Banach spaces and of ordered spaces. In the last section we investigate the properties of functions $\psi_{\omega, q}$ and $\|.\|_{\omega, q},(0<\omega<1$, $q<1)$ related to the Lorentz sequence space. Other posibilities for parameters $\omega$ and $q$ are considered, the inverse Holder inequalities and more variants of the Beckenbach-Dresher inequalities are obtained.

2000 MSC: Primary 26D15; Secondary 46B99.
\end{abstract}

Keywords: inequalities, the Beckenbach-Dresher inequality, concave function, inverse Minkowski's inequality, $\psi$-direct sum, the Lorentz sequence space

\section{Preliminaries}

In the fifties of the previous century the following result was obtained:

Let $1 \leq p \leq 2$ and $x_{i}, y_{i}>0, i=1, \ldots, n$. Then

$$
\frac{\sum_{i=1}^{n}\left(x_{i}+y_{i}\right)^{p}}{\sum_{i=1}^{n}\left(x_{i}+y_{i}\right)^{p-1}} \leq \frac{\sum_{i=1}^{n} x_{i}^{p}}{\sum_{i=1}^{n} x_{i}^{p-1}}+\frac{\sum_{i=1}^{n} y_{i}^{p}}{\sum_{i=1}^{n} y_{i}^{p-1}} .
$$

The above-mentioned discrete inequality was given by Beckenbach [1], and the integral version is due to Dresher [2] (see also [3]). From that time, some generalizations of the Beckenbach-Dresher inequality (1) have appeared. Here, we are pointing out articles of Pečarić and Beesack [4], Petree and Persson [5], Persson [6] and Varošanec [7], where the reader can find related literature about this inequality Here we consider inequalities of Beckenbach-Dresher type in more general structures, namely in $\psi$-direct sums.

In this article we follow definitions and notations from the paper [8]. Let $\Psi$ denote the family of all convex functions $\psi$ on $[0,1]$ with $\psi(0)=\psi(1)=1$ satisfying

$$
\max \{1-t, t\} \leq \psi(t) \leq 1, \quad(0 \leq t \leq 1) .
$$

It is known (see [9]), that $\Psi$ is in one-to-one correspondence with the set $N_{a}$ of all absolute and normalized norms on $\mathbf{C}^{2}$, i.e., such that

$$
\|(x, y)\|=\|(|x|,|y|)\| \text { and }\|(1,0)\|=\|(0,1)\|=1 \text {. }
$$

(c) 2012 Nikolova et al; licensee Springer. This is an Open Access article distributed under the terms of the Creative Commons Attribution License (http://creativecommons.org/licenses/by/2.0), which permits unrestricted use, distribution, and reproduction in any medium, provided the original work is properly cited. 
Namely, if $\|.\| \in N_{a}$ and $\psi(t)=\|(1-t, t)\|$, then $\psi \in \Psi$. Conversely, if $\psi \in \Psi$, then

$$
\|(x, y)\|_{\psi}=\left\{\begin{array}{lr}
(|x|+|y|) \psi\left(\frac{|y|}{|x|+|y|}\right), & (x, y) \neq(0,0) \\
0, & (x, y)=(0,0)
\end{array}\right.
$$

is a norm and $\|\cdot\|_{\psi} \in N_{a}$.

Some examples of convex functions and the corresponding norms are the following:

Example 1. The convex functions $\psi_{p}(t)=\left[(1-t)^{p}+t^{p}\right]^{\frac{1}{p}}, 1 \leq p<\infty, 0 \leq t \leq 1$, correspond to $l_{p}$-norms of $\mathbf{C}^{2}$. For $p=\infty$, the function $\psi_{\infty}(t)=\max \{t, 1-t\}, 0 \leq t \leq 1$, corresponds to the norm $l_{\infty}$.

Example 2. Let

$$
\|(x, y)\|_{\omega, q}=\left(x^{* q}+\omega y^{* q}\right)^{\frac{1}{q}}
$$

where $\left\{x^{*}, y^{*}\right\}$ is the non-increasing rearrangement of $\{|x|,|y|\}$. If $0<\omega<1,1 \leq q$, then $\|\cdot\|_{\omega, q}$ is a norm of the two-dimensional Lorentz sequence space $d^{(2)}(\omega, q)$, it belongs to $N_{a}$ and its dual norm was computed recently in [10]. The corresponding function from $\Psi$ is

$$
\psi_{\omega, q}(t)=\left\{\begin{array}{l}
\left((1-t)^{q}+\omega t^{q}\right)^{\frac{1}{q}}, \text { if } 0 \leq t \leq \frac{1}{2} \\
\left(t^{q}+\omega(1-t)^{q}\right)^{\frac{1}{q}}, \text { if } \frac{1}{2} \leq t \leq 1
\end{array}\right.
$$

If $\omega=2^{\frac{q}{p}-1}$, then we get a classical Lorentz $l_{p, q^{-}}$norm.

Example 3. For $\alpha, 1 / 2 \leq \alpha \leq 1$, let us define the following function

$$
\psi_{\alpha}(t)= \begin{cases}\frac{\alpha-1}{\alpha} t+1, & 0 \leq t \leq \alpha, \\ t, & \alpha \leq t \leq 1 .\end{cases}
$$

$\psi_{\alpha} \in \Psi$ and the corresponding norm is

$$
\|(z, w)\|_{\psi_{\alpha}}=\max \{\|z\|+(2-1 / \alpha)\|w\|,\|w\|\} .
$$

Example 4. Let $1 \leq q<p \leq \infty$ and $2^{\frac{1}{p}-\frac{1}{q}}<\lambda<1$. Let $\psi_{p, q, \lambda}=\max \left\{\psi_{p}, \lambda \psi_{q}\right\}$. Then the corresponding norm is $\|\cdot\|_{p, q, \lambda}=\max \left\{\|\cdot\|_{p}, \lambda\|\cdot\|_{q}\right\}$.

Example 5. Let $1 / 2 \leq \beta \leq 1$ and let $\psi_{\beta}(t)=\max \{1-t, t, \beta\}$ (note that neither $\psi_{\beta} \geq \psi_{2}$ nor $\psi_{\beta} \leq \psi_{2}$.). The corresponding norm is

$$
\|(x, y)\|_{\psi \beta}(t)=\left\{\begin{array}{l}
|y|, \frac{|x|}{|y|} \leq \frac{1-\beta}{\beta}, \\
\beta, \frac{1-\beta}{\beta} \leq \frac{|x|}{|y|} \leq \frac{\beta}{1-\beta}, \\
|x|, \frac{|x|}{|y|} \geq \frac{\beta}{1-\beta} .
\end{array}\right.
$$

For $\psi \in \Psi$ let $\|.\|_{\psi}^{*}$ denote the dual of the norm $\|.\|_{\psi}$. From [11] we have that $\|.\|_{\psi}^{*}$ is an absolute normalized norm and the corresponding convex function $\psi^{*} \in \Psi$ is 


$$
\psi *(t)=\sup _{0 \leq s \leq 1} \frac{(1-s)(1-t)+s t}{\psi(s)}
$$

for $t$ with $0 \leq t \leq 1$. For the norms $\|\cdot\|_{\psi}$ and $\|.\|_{\psi}^{*}$ we have the following Hölder-type inequality:

$$
\left\|\left(x_{1}, y_{1}\right)\right\|_{\psi}\left\|\left(x_{2}, y_{2}\right)\right\|_{\psi *} \geq\left|x_{1} x_{2}\right|+\left|y_{1} y_{2}\right|,
$$

where $x_{1}, x_{2}, y_{1}, y_{2} \in \mathbf{C}$.

Since the proof of Beckenbach-Dresher inequality can be obtained as an application of the Minkowski inequality the Holder inequality and its inverse inequalities in different cases, we are going to see what kind of such inequalities we could prove using some ideas of $\psi$-direct sums. In the following section we consider a family of concave functions $\tilde{\Psi}$. We prove some properties of concave functions and the inverse Minkowski inequality Using these results and combining with the known results about the family $\Psi$ and normalized absolute norms we obtain a variant of the BeckenbachDresher inequality related to those norms. In the third section we are considering $\Psi$ direct sums of Banach and ordered spaces. Finally, the last section is devoted to the two-dimensional Lorentz sequence space and its variants. There we obtain several inequalites of the Hölder type.

\section{A family $\tilde{\Psi}$ of concave functions and a generalization of the Beckenbach- Dresher inequality}

Let $\tilde{\Psi}$ denotes the family of all concave functions $\tilde{\psi}$ on $[0,1]$ with $\tilde{\psi}(0)=\tilde{\psi}(1)=1$. Let us define the map $\|\cdot\|_{\tilde{\psi}}$ on $\mathbf{C}^{2}$ by

$$
\|(z, w)\|_{\tilde{\psi}}= \begin{cases}(|z|+|w|) \tilde{\psi}\left(\frac{|w|}{|z|+|w|}\right), & (z, w) \neq(0,0) \\ 0 & (z, w)=(0,0)\end{cases}
$$

As it is proved in the next proposition for this map $\|\cdot\|_{\tilde{\psi}}$ the inverse Minkowski inequality holds. For that reason we call it a pseudo-norm.

Proposition 6. Let $\tilde{\psi}$ be a concave function on $[0,1]$. Then the inverse Minkowski inequality holds, i.e. for $u, v, z, w \in \mathbf{C}$ the following is valid:

$$
\|(|u|+|z|,|v|+|w|)\|_{\tilde{\psi}} \geq\|(|u|,|v|)\|_{\tilde{\psi}}+\|(|z|,|w|)\|_{\tilde{\psi}} .
$$

Proof. Using concavity of the function $\tilde{\psi}$ and the equality

$$
\frac{|v|+|w|}{|u|+|z|+|v|+|w|}=\frac{|u|+|v|}{|u|+|z|+|v|+|w|} \frac{|v|}{|u|+|v|}+\frac{|z|+|w|}{|u|+|z|+|v|+|w|} \frac{|w|}{|z|+|w|}
$$

we get that

$$
\begin{aligned}
\|(|u|+|z|,|v|+|w|)\|_{\tilde{\psi}} & =(|u|+|z|+|v|+|w|) \tilde{\psi}\left(\frac{|v|+|w|}{|u|+|z|+|v|+|w|}\right) \\
& \geq(|u|+|v|) \tilde{\psi}\left(\frac{|v|}{|u|+|v|}\right)+(|z|+|w|) \tilde{\psi}\left(\frac{|w|}{|z|+|w|}\right) \\
& =\|(|u|,|v|)\|_{\tilde{\psi}}+\|(|z|,|w|)\|_{\tilde{\psi}} .
\end{aligned}
$$

The proof is complete. 
Our next result reads:

Proposition 7. Let $\tilde{\psi}$ be a concave function on $[0,1], \tilde{\psi}(t) \geq 0$. Then $\frac{\tilde{\psi}(t)}{t}$ is nonincreasing on $(0,1]$ and $\frac{\tilde{\psi}(t)}{1-t}$ is non-decreasing on $[0,1)$. If $\tilde{\psi}(t)>0$ then the words non-increasing and non-decreasing are replaced by decreasing and increasing, correspondingly.

Moreover, if $0 \leq p \leq r, 0 \leq q \leq s$, we have that

$$
\|(p, q)\|_{\tilde{\psi}} \leq\|r, s\|_{\tilde{\psi}} .
$$

Proof. Let $0<s<t<1$.

(1) Put $p=t / s$ and write $s=\frac{1}{p} t+\frac{1}{p^{\prime}} 0$ where $\frac{1}{p}+\frac{1}{p^{\prime}}=1$. Then, by concavity of $\tilde{\psi}$, we get that

$$
\tilde{\psi}(s) \geq \frac{1}{p} \tilde{\psi}(t)+\frac{1}{p^{\prime}} \tilde{\psi}(0) \geq \frac{1}{p} \psi(t) .
$$

Hence $\tilde{\psi}(s) \geq \frac{s}{t} \tilde{\psi}(t)$, i.e. $\frac{\tilde{\psi}(s)}{s} \geq \frac{\tilde{\psi}(t)}{t}$. If $\tilde{\psi}(0)>0$, then the inequality is strict.

(2) Let now $p=\frac{1-s}{1-t}$. Then $t=\frac{p-1}{p}+\frac{s}{p}=\frac{1}{p^{\prime}} \cdot 1+\frac{1}{p} \cdot s$. By concavity of $\tilde{\psi}$ we find that

$$
\tilde{\psi}(t) \geq \frac{1}{p} \tilde{\psi}(s)+\frac{1}{p^{\prime}} \tilde{\psi}(1) \geq \frac{1}{p} \tilde{\psi}(s) .
$$

Hence $\tilde{\psi}(t) \geq \frac{1-t}{1-s} \tilde{\psi}(s)$ i.e. $\frac{\tilde{\psi}(t)}{1-t} \geq \frac{\tilde{\psi}(s)}{1-s}$. If $\tilde{\psi}(1)>0$, then the inequality is strict.

To prove the monotonity property we proceed like in [8]. Firstly if $0 \leq p \leq r, 0 \leq q \leq s$, then

$$
\frac{q}{p+q} \geq \frac{q}{r+q} \text { and } \frac{q}{r+q} \leq \frac{s}{r+s} .
$$

Using the fact that $\frac{\tilde{\psi}(t)}{t}$ is non-increasing and $\frac{\tilde{\psi}(t)}{1-t}$ is non-decreasing we obtain that

$$
\begin{aligned}
\|(p, q)\|_{\tilde{\psi}} & =(p+q) \tilde{\psi}\left(\frac{q}{p+q}\right)=q \cdot \frac{\tilde{\psi}\left(\frac{q}{p+q}\right)}{\frac{q}{p+q}} \leq q \cdot \frac{\tilde{\psi}\left(\frac{q}{r+q}\right)}{\frac{q}{r+q}}=(r+q) \tilde{\psi}\left(\frac{q}{r+q}\right) \\
& =r \cdot \frac{\tilde{\psi}\left(\frac{q}{r+q}\right)}{\frac{r}{r+q}} \leq r \cdot \frac{\tilde{\psi}\left(\frac{s}{s+r}\right)}{\frac{r}{s+r}}=(r+s) \tilde{\psi}\left(\frac{s}{r+s}\right)=\|(r, s)\|_{\tilde{\psi}} .
\end{aligned}
$$

The proof is complete.

Let $\psi \in \tilde{\Psi}$. Denote

$$
\psi_{*}(t)=\inf _{0 \leq s \leq 1} \frac{(1-s)(1-t)+s t}{\psi(s)}
$$


for $0 \leq t \leq 1$. The corresponding map $\|.\|_{\psi_{*}}$ is defined by (3). Using similar arguments as in [10] we can prove the following:

Proposition 8. Let $\psi \in \tilde{\Psi}$ be symmetric with respect to $t=1 / 2$. Then $\psi_{*}$ is symmetric with respect to $t=\frac{1}{2}$. Moreover,

$$
\psi_{*}(t)=\inf _{0 \leq s \leq \frac{1}{2}} \frac{(1-s)(1-t)+s t}{\psi(s)}
$$

for all $t$ with $\frac{1}{2} \leq t \leq 1$.

The following inverse Hölder-type inequality holds:

Proposition 9. Let $x_{1}, x_{2}, y_{1}, y_{2} \in \mathrm{C}$. If $\psi \in \tilde{\Psi}$, then

$$
\left\|\left(x_{1}, y_{1}\right)\right\|_{\psi}\left\|\left(x_{2}, y_{2}\right)\right\|_{\psi_{*}} \leq\left|x_{1} x_{2}\right|+\left|y_{1} y_{2}\right| \text {. }
$$

Proof. From the definition of the function $\psi_{*}$ we get that

$$
(1-s)(1-t)+s t \geq \psi(s) \psi_{*}(t) .
$$

Putting in that inequality $s=\frac{\left|y_{1}\right|}{\left|x_{1}\right|+\left|y_{1}\right|}, t=\frac{\left|y_{2}\right|}{\left|x_{2}\right|+\left|y_{2}\right|}$ and using formula (3) we get (5).

Example 10. Let $0<\mathrm{p}<1$. The function $\psi_{p}$ belongs to $\tilde{\Psi}$ and the related function $\psi_{*}$ is $\psi_{q}$, where $\frac{1}{p}+\frac{1}{q}=1$. In this case inequality (5) has a form

$$
\left|x_{1}\right|\left|x_{2}\right|+\left|y_{1}\right|\left|y_{2}\right| \geq\left(\left|x_{1}\right|^{p}+\left|x_{2}\right|^{p}\right)^{\frac{1}{p}}\left(\left|y_{1}\right|^{q}+\left|y_{2}\right|^{q}\right)^{\frac{1}{q}}
$$

which is the reversed Hölder inequality in the simplest form, see e.g., [12, p. 99].

The following result is a variant of the Beckenbach-Dresher inequality, obtained by using the inverse Holder inequality of the concrete space $l_{1 / u}$, the Minkowski inequality for the norm $\|.\|_{\psi}$ and the inverse Minkowski inequality (4).

Theorem 11. Let $\psi \in \Psi, \tilde{\psi} \in \tilde{\Psi}$. Let $f_{1}=\left(a_{1}^{1}, a_{2}^{1}\right), f_{2}=\left(a_{1}^{2}, a_{2}^{2}\right), g_{1}=\left(\left|b_{1}^{1}\right|,\left|b_{2}^{1}\right|\right), g_{2}=\left(\left|b_{1}^{2}\right|,\left|b_{2}^{2}\right|\right)$ where $a_{i^{\prime}}^{j},{ }_{i}^{j} \in \mathbf{C}, i, j=1,2$ and let $\left\|g_{1}\right\|_{\tilde{\psi}} \neq 0,\left\|g_{2}\right\|_{\tilde{\psi}} \neq 0$.

If $u \geq 1$, then

$$
\frac{\left\|f_{1}+f_{2}\right\|_{\psi}^{u}}{\left\|g_{1}+g_{2}\right\|_{\tilde{\psi}}^{u-1}} \leq \frac{\left\|f_{1}\right\|_{\psi}^{u}}{\left\|g_{1}\right\|_{\tilde{\psi}}^{u-1}}+\frac{\left\|f_{2}\right\|_{\psi}^{u}}{\left\|g_{2}\right\|_{\tilde{\psi}}^{u-1}} .
$$

The inequality holds also when $u<0, \psi \in \tilde{\Psi}, \tilde{\psi} \in \Psi$. If $0<u<1, \psi \in \tilde{\Psi}, \tilde{\psi} \in \tilde{\Psi}$, then the inequality holds in the opposite direction.

Proof. To prove this inequality in the first case we use the Minkowski inequality for the norm $\|\cdot\|_{\psi}$, the inverse Minkowski inequality (4) for the pseudo-norm $\|\cdot\|_{\tilde{\psi}}$ and the inverse Hölder inequality for the $l_{1 / u}$-norm:

$$
\begin{aligned}
\frac{\left\|f_{1}+f_{2}\right\|_{\psi}^{u}}{\left\|g_{1}+g_{2}\right\|_{\tilde{\psi}}^{u-1}} & \leq\left(\left\|f_{1}\right\|_{\psi}+\left\|f_{2}\right\|_{\psi}\right)^{u}\left(\left\|g_{1}\right\|_{\tilde{\psi}}+\left\|g_{2}\right\|_{\tilde{\psi}}\right)^{1-u} \\
& \leq\left\|f_{1}\right\|_{\psi}^{u}\left\|g_{1}\right\|_{\tilde{\psi}}^{1-u}+\left\|f_{2}\right\|_{\psi}^{u}\left\|g_{2}\right\|_{\tilde{\psi}}^{1-u}=\frac{\left\|f_{1}\right\|_{\psi}^{u}}{\left\|g_{1}\right\|_{\tilde{\psi}}^{u-1}}+\frac{\left\|f_{2}\right\|_{\psi}^{u}}{\left\|g_{2}\right\|_{\tilde{\psi}}^{u-1}} .
\end{aligned}
$$




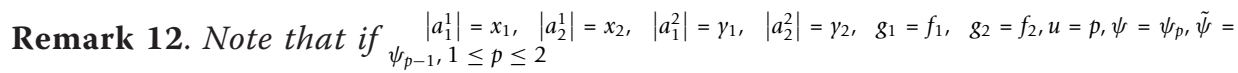
we get the classical Beckenbach inequality (1) for $n=2$.

\section{$3 \Psi$-Direct sums of spaces and some more generalizations of the Beckenbach-Dresher inequality}

The $\psi$-direct sum $X \oplus_{\psi} Y$ of the Banach spaces $X$ and $Y$ is a direct sum $X \oplus_{\psi} Y$ equipped with the norm $\|(x, y)\|_{\psi}=\left\|\left(\|x\|_{X},\|y\|_{Y}\right)\right\|_{\psi}$. This extends the notion of the $l_{p^{-}}$ sum $X \oplus_{p} Y$. Recently various geometric properties of $\psi$ - direct sums have been investigated by many authors [11,13-18].

In the $\psi$-direct sum $X \oplus_{\psi} Y$ of Banach spaces the Minkowski inequality holds, i.e., we have the following:

Let $A$ and $C$ be Banach spaces and let $f_{1}=\left(a_{1}^{1}, a_{2}^{1}\right), f_{2}=\left(a_{1}^{2}, a_{2}^{2}\right), a_{1}^{i} \in A, a_{2}^{i} \in C, i=1,2$. Then

$$
\left\|f_{1}+f_{2}\right\|_{A \oplus_{\psi} C} \leq\left\|f_{1}\right\|_{A \oplus_{\psi} C}+\left\|f_{2}\right\|_{A \oplus_{\psi} C} .
$$

Let us improve that idea to the sum of ordered spaces.

Let $B$ and $D$ be ordered spaces equiped with pseudo-norms $\|\cdot\|_{B}$ and $\|\cdot\|_{D}$ and let $g_{1}=\left(\left|b_{1}^{1}\right|,\left|b_{2}^{1}\right|\right), g_{2}=\left(\left|b_{1}^{2}\right|, \mid b_{2}^{2}\right) \mid, b_{1}^{i} \in B, b_{2}^{i} \in D, i=1,2$. That means that in $B$ and $D$ the inverse Minkowski inequality holds, i.e.,

$$
\left\|\left|b_{1}^{1}\right|+\left|b_{1}^{2}\right|\right\|_{B} \geq\left\|b_{1}^{1}\right\|_{B}+\left\|b_{1}^{2}\right\|_{B^{\prime}}\left\|\left|b_{2}^{1}\right|+\left|b_{2}^{2}\right|\right\|_{D} \geq\left\|b_{2}^{1}\right\|_{D}+\left\|b_{2}^{2}\right\|_{D} .
$$

Let $\tilde{\psi}$ be a concave function from $\tilde{\Psi}$. Let us define $B \oplus_{\tilde{\psi}} D$. $B \oplus_{\tilde{\psi}} D$ is called $\tilde{\psi}$-direct sum of spaces $B$ and $D$. Using monotonicity of the pseudo-norm $\|\cdot\|_{\tilde{\Psi}}$ generated by the function $\tilde{\psi}$ and the fact that the inverse Minkowski inequality holds for this pseudo-norm, we get the following estimates for the $\tilde{\psi}$-direct sum:

$$
\begin{aligned}
\left\|g_{1}+g_{2}\right\|_{B \oplus_{\tilde{\psi}} D} & =\left\|\left(\left\|\left|b_{1}^{1}\right|+\left|b_{1}^{2}\right|\right\|_{B^{\prime}}\left\|\left|b_{2}^{1}\right|+\left|b_{2}^{2}\right|\right\|_{D}\right)\right\|_{\tilde{\psi}} \\
& \geq\left\|\left(\left\|b_{1}^{1}\right\|_{B}+\left\|b_{1}^{2}\right\|_{B^{\prime}}\left\|b_{2}^{1}\right\|_{D}+\left\|b_{2}^{2}\right\|_{D}\right)\right\|_{\tilde{\psi}}=\left\|\left(\left\|b_{1}^{1}\right\|_{B^{\prime}}\left\|b_{2}^{1}\right\|_{D}\right)+\left(\left\|b_{1}^{2}\right\|_{B^{\prime}}\left\|b_{2}^{2}\right\|_{D}\right)\right\|_{\tilde{\psi}} \\
& \geq\left\|\left(\left\|b_{1}^{1}\right\|_{B^{\prime}}\left\|b_{2}^{1}\right\|_{D}\right)\right\|_{\tilde{\psi}}+\left\|\left(\left\|b_{1}^{2}\right\|_{B^{\prime}}\left\|b_{2}^{2}\right\|_{D}\right)\right\|_{\tilde{\psi}}=\left\|g_{1}\right\|_{B \oplus_{\tilde{\psi}} D}+\left\|g_{2}\right\|_{B \oplus_{\tilde{\psi}} D^{\prime}}
\end{aligned}
$$

So we have showed that when $\tilde{\psi} \in \tilde{\Psi}$ and the inverse Minkowski inequality holds in $B$ and $D$, then this inequality holds also for the pseudo-norm of $B \oplus_{\tilde{\psi}} D$.

Our next result is the following inequalities of the Beckenbach-Dresher type:

Theorem 13. Let $\psi \in \Psi, \tilde{\psi} \in \tilde{\Psi}$ and $A, C$ be Banach spaces, B, D ordered spaces such that the inverse Minkowski inequality holds. Let $f_{1}=\left(a_{1}^{1}, a_{2}^{1}\right), f_{2}=\left(a_{1}^{2}, a_{2}^{2}\right), g_{1}=\left(\left|b_{1}^{1}\right|,\left|b_{2}^{1}\right|\right), g_{2}=\left(\left|b_{1}^{2}\right|,\left|b_{2}^{2}\right|\right),\left\|g_{1}\right\|_{\tilde{\psi}} \neq 0,\left\|g_{2}\right\|_{\tilde{\psi}} \neq 0, \quad$ for $a_{1}^{i} \in A, a_{2}^{i} \in C, \quad b_{1}^{i} \in B, \quad b_{2}^{i} \in D, \quad i=1,2$. If $u \geq 1$, then

$$
\frac{\left\|f_{1}+f_{2}\right\|_{A \oplus_{\psi} C}^{u}}{\left\|g_{1}+g_{2}\right\|_{B \oplus_{\tilde{\psi}} D}^{u-1}} \leq \frac{\left\|f_{1}\right\|_{A \oplus_{\psi} C}^{u}}{\left\|g_{1}\right\|_{B \oplus_{\tilde{\psi}} D}^{u-1}}+\frac{\left\|f_{2}\right\|_{A \oplus_{\psi} C}^{u}}{\left\|g_{2}\right\|_{B \oplus_{\tilde{\psi}} D}^{u-1} .}
$$

Proof. The proof is similar to the proof of Theorem 11 so we omit the details.

Remark 14. If $u<1$, the analogue result can be considered. 
If $g_{1}=f_{1}, g_{2}=f_{2}, u=p, \psi=\psi_{p}, \tilde{\psi}=\psi_{p-1}, 1 \leq p \leq 2$, then we get that

$$
\frac{\left\|a_{1}^{1}+a_{1}^{2}\right\|_{A}^{p}+\left\|a_{2}^{1}+a_{2}^{2}\right\|_{C}^{p}}{\left\|\left|b_{1}^{1}\right|+\left|b_{1}^{2}\right|\right\|_{B}^{p-1}+\left\|\left|b_{2}^{1}\right|+\left|b_{2}^{2}\right|\right\|_{D}^{p-1}} \leq \frac{\left\|a_{1}^{1}\right\|_{A}^{p}+\left\|a_{2}^{1}\right\|_{C}^{p}}{\left\|b_{1}^{1}\right\|_{B}^{p-1}+\left\|b_{2}^{1}\right\|_{D}^{p-1}}+\frac{\left\|a_{1}^{2}\right\|_{A}^{p}+\left\|a_{2}^{2}\right\|_{C}^{p}}{\left\|b_{1}^{2}\right\|_{B}^{p-1}+\left\|b_{2}^{1}\right\|_{D}^{p-1}} .
$$

Note that if we take $A=B=C=D=\mathbf{R}$ and put $\left|a_{1}^{1}\right|=x_{1},\left|a_{2}^{1}\right|=x_{2},\left|a_{1}^{2}\right|=y_{1},\left|a_{2}^{2}\right|=y_{2}$ then we get the classical Beckenbach inequality (1) for $n=2$.

A natural question arises : can we get some similar generalization of BeckenbachDresher inequality for $n>2$ ?

We use the construction from [19]. Let $\Delta_{n}$ be a set $\Delta_{n}=\left\{\left(s_{1}, \ldots, s_{n-1}\right) \in \mathbf{R}^{n-1}: s_{1}+\ldots\right.$ $\left.+s_{n-1} \leq 1, s_{i} \geq 0,1 \leq i \leq n-1\right\}$. In [19] the authors considered the family $\Psi_{n}$ of all continuous convex functions $\psi$ on $\Delta_{n}$ which satisfy:

$$
\begin{aligned}
& \psi(0,0, \ldots, 0)=\psi(0,1,0, \ldots, 0)=\cdots=\psi(0,0, \ldots, 0,1)=1 . \\
& \psi\left(s_{1}, \ldots, s_{n-1}\right) \geq\left(s_{1}+\cdots+s_{n-1}\right) \psi\left(\frac{s_{1}}{s_{1}+\cdots+s_{n-1}}, \ldots, \frac{s_{n-1}}{s_{1}+\cdots+s_{n-1}}\right) \\
& \psi\left(s_{1}, \ldots, s_{n-1}\right) \geq\left(1-s_{k}\right) \psi\left(\frac{s_{1}}{1-s_{k}}, \ldots, \frac{s_{k-1}}{1-s_{k}}, 0, \frac{s_{k+1}}{1-s_{k}}, \ldots, \frac{s_{n-1}}{1-s_{k}}\right)
\end{aligned}
$$

for $k=1, \ldots, n-1$. They showed that the family $A N_{n}$ of all absolute normalized norms on $\mathbf{C}^{n}$ and the family $\Psi_{n}$ are in one-to-one correspondence: if $\|.\| \in A N_{n}$, then

$$
\psi\left(s_{1}, \ldots, s_{n-1}\right)=\left\|\left(1-\sum_{i=1}^{n-1} s_{i}, s_{1}, \ldots, s_{n-1}\right)\right\|
$$

belongs to $\Psi_{n}$ and for given $\psi \in \Psi_{n}$ the following norm $\|\cdot\|_{\psi}$ belongs to $A N_{n}$ :

$$
\left\|\left(z_{1}, \ldots, z_{n}\right)\right\|_{\psi}=\left(\left|z_{1}\right|+\ldots+\left|z_{n}\right|\right) \psi\left(\frac{\left|z_{2}\right|}{\left|z_{1}\right|+\ldots+\left|z_{n}\right|}, \ldots, \frac{\left|z_{n}\right|}{\left|z_{1}\right|+\ldots+\left|z_{n}\right|}\right)
$$

if $\left(z_{1}, \ldots, z_{n}\right) \neq(0, \ldots, 0)$ and $\|(0, \ldots, 0)\|_{\psi}=0$.

The set $\tilde{\Psi}_{n}$ is defined as a set of all positive continuous concave functions $\tilde{\psi}$ on $\Delta_{n}$, which satisfy condition (6).

Let us define the pseudo-norm $\|\cdot\|_{\tilde{\psi}}$ by the formula given in (7). Consider for simplicity the case $n=3$. If the function $\tilde{\psi}$ is concave it is not difficult to prove using the same idea as in Proposition 7, that, for $\lambda>1$, it yields that

$$
\frac{\tilde{\psi}(s, t)}{s} \geq \frac{\tilde{\psi}(\lambda s, \lambda t)}{\lambda s} \text { and } \frac{\tilde{\psi}(s, t)}{1-s} \geq \frac{\tilde{\psi}(\lambda s, \lambda t)}{1-\lambda s} .
$$

In the same way as it was done in Propositions 6 and 7 we can prove monotonicity of the pseudonorm and the inverse Minkowski inequality

$$
\left\|\left|\left(\left|x_{1}\right|,\left|y_{1}\right|,\left|z_{1}\right|\right)+\left(\left|x_{2}\right|,\left|y_{2}\right|,\left|z_{2}\right|\right)\left\|_{\tilde{\psi}} \geq\right\|\right|\left(\left|x_{1}\right|,\left|y_{1}\right|,\left|z_{1}\right|\right)\right\|_{\tilde{\psi}}+\| \mid\left(\left(\left|x_{2}\right|,\left|y_{2}\right|,\left|z_{2}\right|\right) \|_{\tilde{\psi}} .\right.
$$

As above we could prove that if $B_{1}, \ldots, B_{n}$ have inverse Minkowski property and $\tilde{\psi} \in \tilde{\Psi}_{n}$, then $\left(B_{1} \oplus B_{2} \oplus \ldots \oplus B_{n}\right)_{\tilde{\psi}}$ would have inverse Minkowski property. So we can state the following generalization of the previous Theorem: 
Theorem 15. Let $\psi \in \Psi_{n}, \tilde{\psi} \in \tilde{\Psi}_{n}$ and $A_{1}, A_{2}, \ldots, A_{n}$ be Banach spaces, $B_{1}, B_{2}, \ldots, B_{n}$ be ordered spaces in which the inverse Minkowski inequality hold. Let $f_{1}=\left(a_{1}^{1}, a_{2}^{1}, \ldots a_{n}^{1}\right), f_{2}=\left(a_{1}^{2}, a_{2}^{2}, \ldots, a_{n}^{2}\right), g_{1}=\left(\left|b_{1}^{1}\right|,\left|b_{2}^{1}\right|, \ldots,\left|b_{n}^{1}\right|\right), g_{2}=\left(\left|b_{1}^{2}\right|,\left|b_{2}^{2}\right|, \ldots,\left|b_{n}^{2}\right|\right),\left\|g_{1} \mid\right\|_{\tilde{\psi}} \neq, \quad$ where $0,\left\|g_{2}\right\|_{\bar{\psi}} \neq 0$

$a_{i}^{1}, a_{i}^{2} \in A_{i}, b_{i}^{1}, b_{i}^{2} \in B_{i}$ for $i=1,2, \ldots, n$. If $u \geq 1$, then

$$
\frac{\left\|f_{1}+f_{2}\right\|_{\left(A_{1} \oplus A_{2} \oplus \ldots \oplus A_{n}\right)_{\psi}}^{u}}{\left\|g_{1}+g_{2}\right\|_{\left(B_{1} \oplus B_{2} \oplus \ldots \oplus B_{n}\right)_{\tilde{\psi}}}^{u-1}} \leq \frac{\left\|f_{1}\right\|_{\left(A_{1} \oplus A_{2} \oplus \ldots \oplus A_{n}\right)_{\psi}}^{u}}{\left\|g_{1}\right\|_{\left(B_{1} \oplus B_{2} \oplus \ldots \oplus B_{n}\right)_{\tilde{\psi}}}^{u-1}}+\frac{\left\|f_{2}\right\|_{\left(A_{1} \oplus A_{2} \oplus \ldots \oplus B_{n}\right)_{\psi}}^{u}}{\left\|g_{2}\right\|_{\left(B_{1} \oplus B_{2} \oplus \ldots \oplus B_{n}\right)_{\tilde{\psi}}}^{u-1}} .
$$

Proof. The proof is completely similar as that before, so we leave out the details.

\section{Norm of the Lorentz sequence space and its variants}

Let us consider two-dimensional Lorentz sequence space $d^{(2)}(\omega, q)$, where $0<\omega<1,1$ $\leq q$. It is $\mathbf{R}^{2}$ with the norm

$$
\|(x, y)\|_{\omega, q}=\left(x^{* q}+\omega y^{* q}\right)^{\frac{1}{q}} .
$$

The corresponding convex function is

$$
\psi_{\omega, q}(t)=\left\{\begin{array}{l}
\left((1-t)^{q}+\omega t^{q}\right)^{\frac{1}{q}}, 0 \leq t \leq \frac{1}{2} \\
\left(t^{q}+\omega(1-t)^{q}\right)^{\frac{1}{q}}, \frac{1}{2} \leq t \leq 1 .
\end{array}\right.
$$

The dual norm of $d^{(2)}(\omega, q)$ is completely determined by Mitani and Saito in [10] by finding the corresponding function $\psi_{\omega, q}^{*}$. Namely, if $0<\omega<1$ and $1<q<\infty$, then we have that

$$
\psi_{\omega, q}^{*}(t)= \begin{cases}\left((1-t)^{p}+\omega^{1-p} t^{p}\right)^{\frac{1}{p}}, & 0 \leq t \leq \frac{\omega}{1+\omega} \\ (1+\omega)^{\frac{1}{p}-1}, & \frac{\omega}{1+\omega} \leq t \leq \frac{1}{1+\omega} \\ \left(t^{p}+\omega^{1-p}\left((1-t)^{p}\right)^{\frac{1}{p}},\right. & \frac{1}{1+\omega} \leq t \leq 1\end{cases}
$$

where $\frac{1}{p}+\frac{1}{q}=1$. The dual norm is equal to

$$
\|(x, y)\|_{\omega, q}^{*}=\|(x, y)\|_{\psi_{\omega, q}^{*}}=\left\{\begin{array}{l}
\left(|x|^{p}+\omega^{1-p}|y|^{p}\right)^{\frac{1}{p}}, \quad \omega|x| \geq|y|, \\
(1+\omega)^{\frac{1}{p}-1}(|x|+|y|), \quad \omega|x| \leq|y| \leq \omega^{-1}|x|, \\
\left(|y|^{p}+\omega^{1-p}|x|^{p}\right)^{\frac{1}{p},} \quad \omega^{-1}|x| \leq|y| .
\end{array}\right.
$$

If $0<\omega<1$ and $q=1$, then

$$
\psi_{\omega, 1}^{*}(t)=\left\{\begin{array}{l}
1-t, \quad 0 \leq t \leq \frac{\omega}{1+\omega} \\
\frac{1}{1+\omega}, \frac{\omega}{1+\omega} \leq t \leq \frac{1}{1+\omega} \\
t, \quad \frac{1}{1+\omega} \leq t \leq 1
\end{array}\right.
$$


and

$$
\|(x, y)\|_{\omega, 1}^{*}=\|(x, y)\|_{\psi_{\omega, q}^{*}}= \begin{cases}|x|, & \omega|x| \geq|y| \\ \frac{1}{1+\omega}(|x|+|y|), & \omega|x| \leq|y| \leq \omega^{-1}|x| \\ |y|, & \omega^{-1}|x| \leq|y| .\end{cases}
$$

If $0<q<1, \omega>1$, then $\psi_{\omega, q}$ is a concave function from $\tilde{\Psi}$ and we have the inverse Minkowski inequality for the corresponding pseudo-norm. Indeed, if $0 \leq t \leq 1 / 2$, then the function is increasing and concave; if $1 / 2 \leq t \leq 1$, then it is decreasing and concave (which can be shown by finding the first and second derivatives). Let $0 \leq t_{1} \leq 1 / 2 \leq t_{2} \leq 1$. Consider the line connecting the points $\left(t_{1}, \psi_{\omega, q}\left(t_{1}\right)\right)$ and $\left(t_{2}, \psi_{\omega, q}\left(t_{2}\right)\right)$. For the concavity it is enough to show that the graph of the function is above this line. In fact, this is the case, because if we consider for instance $t_{1} \leq t \leq 1 / 2$, then (because of concavity on this interval) the graph is above the line connecting the points $\left(t_{1}, \psi_{\omega, q}\left(t_{1}\right)\right)$ and $\left(1 / 2, \psi_{\omega, q}(1 / 2)\right)$.

Lemma 1 from [20] for the case $1 \leq q<\infty, 0<\omega \leq 1$ asserts that

$$
\left(\frac{\omega+1}{2}\right)^{\frac{1}{q}}\|a\|_{q} \leq\|a\|_{\omega, q} \leq\|a\|_{q}
$$

for all $a \in \mathbf{R}^{2}$. It is easy to obtain similar result for other posibilities of parameters $q$ and $\omega$. For example the following holds:

Lemma 16. If $\omega \geq 1$ and $q>0$, then

$$
\|a\|_{q} \leq\|a\|_{\omega, q} \leq\left(\frac{\omega+1}{2}\right)^{\frac{1}{q}}\|a\|_{q}
$$

for all $a \in \mathbf{R}^{2}$.

Our next result reads:

Theorem 17. Let $a, b \in \mathbf{R}^{2}$. Then the following inequality holds

$$
\|a+b\|_{\omega, q} \leq C\left(\|a\|_{\omega, q}+\|b\|_{\omega, q}\right)
$$

where

$$
C=\left\{\begin{array}{l}
1, \quad q \geq 1, \quad 0<\omega \leq 1, \\
\left(\frac{\omega+1}{2}\right)^{\frac{1}{q}}, \quad q \geq 1, \quad 1 \leq \omega, \\
\frac{1}{2}\left(\frac{4}{\omega+1}\right)^{\frac{1}{q}}, \quad 0<q<1, \quad 0<\omega \leq 1, \\
\frac{1}{2}(\omega+1)^{\frac{1}{q}}, \quad 0<q<1, \quad \omega \geq 1 .
\end{array}\right.
$$

Proof. If $q \geq 1,0<\omega \leq 1$, then Kato and Maligranda proved that $C=1$ in [20].

Let $q \geq 1,1 \leq \omega$. Using (9) and the Minkowski inequality for the norm $\|\cdot\|_{q}$ we have that

$$
\begin{aligned}
\|a+b\|_{\omega, q} & \leq\left(\frac{\omega+1}{2}\right)^{\frac{1}{q}}\|a+b\|_{q} \leq\left(\frac{\omega+1}{2}\right)^{\frac{1}{q}}\left(\|a\|_{q}+\|b\|_{q}\right) \\
& \leq\left(\frac{\omega+1}{2}\right)^{\frac{1}{q}}\left(\|a\|_{\omega, q}+\|b\|_{\omega, q}\right) .
\end{aligned}
$$


Let $0<q<1,0<\omega \leq 1$. Using inequality between means of order $q$ and 1 and superadditivity of the function $f(s)=s^{q}$ we have the following:

$$
\begin{aligned}
2\|a+b\|_{q} & =2\left(\left(a_{1}+b_{1}\right)^{q}+\left(a_{2}+b_{2}\right)^{q}\right)^{\frac{1}{q}} \leq\left(2\left(a_{1}+b_{1}+a_{2}+b_{2}\right)^{q}\right)^{\frac{1}{q}} \\
& \leq 2^{\frac{1}{q}}\left(\left(a_{1}^{q}+a_{2}\right)^{\frac{1}{q}}+\left(b_{1}^{q}+b_{2}^{q}\right)^{\frac{1}{q}}\right)=2^{\frac{1}{q}}\left(\|a\|_{q}+\|b\|_{q}\right),
\end{aligned}
$$

where $a=\left(a_{1}, a_{2}\right)$ and $b=\left(b_{1}, b_{2}\right)$.

Combining the inequality $\|a+b\|_{q} \leq 2^{\frac{1}{q}-1}\left(\|a\|_{q}+\|b\|_{q}\right)$ and (8) we get that

$$
\begin{aligned}
\|a+b\|_{\omega, q} & \leq\|a+b\|_{q} \leq 2^{\frac{1}{q}-1}\left(\|a\|_{q}+\|b\|_{q}\right) \\
& \leq 2^{\frac{1}{q}-1}\left(\frac{2}{\omega+1}\right)^{\frac{1}{q}}\left(\|a\|_{\omega, q}+\|b\|_{\omega, q}\right) \\
& =\frac{1}{2}\left(\frac{4}{\omega+1}\right)^{\frac{1}{q}}\left(\|a\|_{\omega, q}+\|b\|_{\omega, q}\right) .
\end{aligned}
$$

Finally, let $0<q<1, \omega \geq 1$. Using the above-proved inequality and (9) we have that

$$
\begin{aligned}
\|a+b\|_{\omega, q} \leq\left(\frac{\omega+1}{2}\right)^{\frac{1}{q}}\|a+b\|_{q} & \leq 2^{\frac{1}{q}-1}\left(\frac{\omega+1}{2}\right)^{\frac{1}{q}}\left(\|a\|_{q}+\|b\|_{q}\right) \\
& \leq \frac{1}{2}(\omega+1)^{\frac{1}{q}}\left(\|a\|_{\omega, q}+\|b\| \omega, q\right) .
\end{aligned}
$$

The proof is complete.

Remark 18. For the second case we see that the quasi-norm constant $C$ is less than or equal to $((\omega+1) / 2)^{\frac{1}{q}}$. We will compare with the result from [21], Proposition 1, where $\omega=2^{\frac{q}{p}-1}, q>q \geq 1$ and the quasi-norm constant is $2^{\frac{1}{p} \text {. Since }}((\omega+1) / 2)^{\frac{1}{q}}<2^{\frac{1}{p}}$ we see that quasi-norm constant $C$ obtained in this theorem is strictly less than the known constant for that case.

Remark 19. As we already mentioned, the norm of dual space of the space $d^{(2)}(\omega, q)$, $q \geq 1,0<\omega \leq 1$, is given in [10].

In the next part of this section we calculate the function $\psi_{* *}^{*}, q$ for $0<q<1$ and $1 \leq$ $\omega$ and the corresponding mapping $\|.\|_{\psi_{*}}$ Using those results we obtain examples of the inverse Holder inequality, and get some new variations of the Beckenbach-Dresher inequality.

Proposition 20. If $0<q<1,1 \leq \omega$, then

$$
\psi_{* \omega, q}(t)= \begin{cases}\left(t^{p}+\omega^{1-p}(1-t)^{p}\right)^{\frac{1}{p}}, & 0<t \leq \frac{1}{\omega+1}, \\ (1+\omega)^{\frac{1}{p}-1}, & \frac{1}{\omega+1} \leq t \leq \frac{\omega}{\omega+1}, \\ \left((1-t)^{p}+\omega^{1-p} t^{p}\right)^{\frac{1}{p}}, & \frac{\omega}{\omega+1} \leq t<1,\end{cases}
$$

where $\frac{1}{p}+\frac{1}{q}=1$ 
Proof. We consider the function

$$
f(s)=f(s, t)=\frac{(1-s)(1-t)+s t}{\psi_{\omega, q}(s)}
$$

for fixed $t$. Let first $t \in\left[\frac{1}{2}, 1\right)$. Here $\psi_{* \omega, q}(t)=\min _{s \in\left[0, \frac{1}{2}\right]} f(s)$.

The derivative of $f$ is

$$
f^{\prime}(s)=\left((1-s)^{q}+\omega s^{q}\right)^{-\frac{1}{q}-1}\left((1-s)^{q-1} t-\omega s^{q-1}(1-t)\right)
$$

and $f\left(s_{0}\right)=0$ when

$$
s_{0}=\frac{t^{p-1}}{t^{p-1}+\omega^{p-1}(1-t)^{p-1}} .
$$

If $\frac{\omega}{\omega+1} \leq t \leq 1$, then $0 \leq s_{0} \leq 1 / 2$. Therefore it is easy to see that the function $f$ attains its minimum at $s=s_{0}$ i.e.,

$$
\psi_{* \omega, q}(t)=f\left(s_{0}\right)=\frac{\left(1-s_{0}\right)(1-t)+s_{0} t}{\left(\left(1-s_{0}\right)^{q}+\omega s_{0}^{q}\right)^{\frac{1}{q}}}=\left((1-t)^{p}+\omega^{1-p} t^{p}\right)^{\frac{1}{p}} .
$$

If $1 / 2 \leq t \leq \frac{\omega}{\omega+1}$, then $s_{0} \geq 1 / 2$. Hence the minimum of $f$ is at $s=1 / 2$ i.e., $\psi_{* \omega, q}(t)=f\left(\frac{1}{2}\right)=(1+\omega)^{\frac{1}{p}-1}$.

Having in mind the symmetry of the function $\psi_{* \omega, q}(t)$ we can end the proof.

In the previous Proposition we consider $t \in(0,1)$ since for $q \in(0,1) p$ is negative. But, it is easy to see that $\psi_{* \omega, q}(0)=\psi_{* \omega, q}(1)=1$.

Proposition 21. If $0<q \leq 1,1 \leq \omega$, then

$$
\|(x, y)\| \psi_{* \omega, q}=\left\{\begin{array}{cc}
\left(\gamma^{* p}+\omega^{(1-p)} x^{* p}\right)^{\frac{1}{p}} & \frac{x *}{y_{*}} \geq \omega, \\
(\omega+1)^{\frac{1}{p}-1}(|x|+|y|) & \frac{x *}{\gamma *} \leq \omega .
\end{array}\right.
$$

Proof. Let $x^{*} \geq \omega y^{*}$. Without loosing of generality, put $x^{*}=|x|$. This means that $|x|$ $\geq \omega|y|$, and then $t=\frac{|y|}{|x|+|y|} \leq \frac{|y|}{\omega|y|+|y|}=\frac{1}{1+\omega}$ and

$$
\begin{aligned}
\|(x, y)\|_{\psi_{* \omega, q}} & =(|x|+|y|)\left(\frac{|y|^{p}}{(|x|+|y|)^{p}}+\omega^{(1-p)} \frac{|x|^{p}}{(|x|+|y|)^{p}}\right)^{\frac{1}{p}} \\
& =\left(|y|^{p}+\omega^{(1-p)}|x|^{p}\right)^{\frac{1}{p}}=\left(\gamma^{* p}+\omega^{(1-p)} x^{* p}\right)^{\frac{1}{p}} .
\end{aligned}
$$

The case when $x^{*}=|y|$ is quite analogue. Let $x^{*} \leq \omega y^{*}$. Let for instance $x^{*}=|x|$ i.e., $\mid$ $y|\leq| x|\leq \omega| y \mid$. Then $\frac{1}{2} \geq t=\frac{|y|}{|x|+|y|} \geq \frac{|y|}{\omega|y|+|y|}=\frac{1}{1+\omega}$ and

$$
\|(x, y)\|_{\psi_{* \omega, q}}=(\omega+1)^{\frac{1}{p}-1}(|x|+|y|) .
$$


Using the inverse Hölder inequality we obtain the following Corollary:

Corollary 22. Let $0<q \leq 1,1 \leq \omega, x_{1}, x_{2}, y_{1}, y_{2}>0$.

If $\frac{x_{2}^{*}}{y_{2}^{*}} \geq \omega$, we have that

$$
\left(x_{1}^{* q}+\omega y_{1}^{* q}\right)^{\frac{1}{q}}\left(y_{2}^{* p}+\omega^{(1-p)} x_{2}^{* p}\right)^{\frac{1}{p}} \leq x_{1} x_{2}+y_{1} y_{2} .
$$

If $\frac{x_{2}^{*}}{y_{2}^{*}} \leq \omega$, we find that

$$
\left(x_{1}^{* q}+\omega y_{1}^{* q}\right)^{\frac{1}{q}}(\omega+1)^{\frac{1}{p}-1}\left(\left|x_{2}\right|+\left|y_{2}\right|\right) \leq x_{1} x_{2}+y_{1} y_{2} .
$$

Let $f_{i}, g_{i}$ be as in Theorem 13, let $f_{1}^{*}, f_{2}^{*}$ be such that $f_{1}^{*}=f_{1}$ if $\left\|f_{1}\right\|_{\psi} \geq\left\|f_{2}\right\|_{\psi}$ and $f_{1}^{*}=f_{2}$ if $\left\|f_{1}\right\|_{\psi} \leq\left\|f_{2}\right\|_{\psi}$. Moreover, let $g_{1}^{*}, g_{2}^{*}$ be such that $g_{1}^{*}=g_{1}$ if $\left\|g_{1}\right\|_{\tilde{\psi}} \geq\left\|g_{2}\right\|_{\tilde{\psi}^{\prime}} g_{1}^{*}=g_{2}$ if $\left\|g_{1}\right\|_{\tilde{\psi}} \leq\left\|g_{2}\right\|_{\tilde{\psi}}$. Hence $\left\|g_{1}^{*}\right\|_{\tilde{\psi}} \geq\left\|g_{2}^{*}\right\|_{\tilde{\psi}}$.

We state the following new variant of the Beckenbach-Dresher inequality:

Theorem 23. Let $u \geq 1,1 \leq \omega$. Let $\psi \in \Psi, \tilde{\psi} \in \tilde{\Psi}, f_{i}, g_{i}$ be as in Theorem 13 and

$$
\begin{aligned}
\left\|g_{1}^{*}\right\|_{\tilde{\psi}} \geq \omega^{\frac{1}{u-1}}\left\|g_{2}^{*}\right\|_{\tilde{\psi}}>0 . \text { Then } \\
\quad \frac{\left\|f_{1}^{*}+\omega f_{2}^{*}\right\|_{\psi}^{u}}{\left\|g_{1}^{*}+\omega^{\frac{u}{u-1}} g_{2}^{*}\right\|_{\tilde{\psi}}^{u-1}} \leq \frac{\left\|f_{1}\right\|_{\psi}^{u}}{\left\|g_{1}\right\|_{\tilde{\psi}}^{u-1}}+\frac{\left\|f_{2}\right\|_{\psi}^{u}}{\left\|g_{2}\right\|_{\tilde{\psi}}^{u-1}} .
\end{aligned}
$$

Proof. Using the Minkowski inequality for the norm $\|\cdot\|_{\psi}$, inverse Minkowski inequality for $\|\cdot\|_{\tilde{\psi}}$ and inverse Hölder inequality i.e., previous corollary for

$$
\begin{gathered}
\frac{\left\|f_{1}^{*}+\omega f_{2}^{*}\right\|_{\psi}^{u}}{\left\|g_{1}^{*}+\omega^{\frac{u}{u-1}} g_{2}^{*}\right\|_{\tilde{\psi}}^{u-1}} \leq\left(\left\|f_{1}^{*}\right\|_{\psi}+\omega\left\|f_{2}^{*}\right\|_{\psi}\right)^{u}\left(\left\|g_{1}^{*}\right\|_{\tilde{\psi}}+\omega^{\frac{u}{u-1}}\left\|g_{2}^{*}\right\|_{\tilde{\psi}}\right)^{1-u}=\left(x_{1}^{* q}+\omega y_{1}^{* q}\right)^{\frac{1}{q}}\left(y_{2}^{* p}+\omega^{(1-p)} x_{2}^{* p}\right)^{\frac{1}{p}} \\
\leq x_{1} x_{2}+\gamma_{1} \gamma_{2}=\left\|f_{1}\right\|_{\psi}^{u}\left\|g_{1}\right\|_{\tilde{\psi}}^{1-u}+\left\|f_{2}\right\|_{\tilde{\psi}}^{u}\left\|g_{2}\right\|_{\tilde{\psi}}^{1-u}=\frac{\left\|f_{1}\right\|_{\psi}^{u}}{\left\|g_{1}\right\|_{\tilde{\psi}}^{u-1}}+\frac{\left\|f_{2}\right\|_{\psi}^{u}}{\left\|g_{2}\right\|_{\tilde{\psi}}^{u-1}} . \\
\frac{\left\|f_{1}^{*}+\omega f_{2}^{*}\right\|_{\psi}^{u}}{\left\|g_{1}^{*}+\omega^{\frac{u}{u-1}} g_{2}^{*}\right\|_{\tilde{\psi}}^{u-1}} \leq\left(\left\|f_{1}^{*}\right\|_{\psi}+\omega\left\|f_{2}^{*}\right\|_{\psi}\right)^{u}\left(\left\|g_{1}^{*}\right\|_{\tilde{\psi}}+\omega^{\frac{u}{u-1}}\left\|g_{2}^{*}\right\|_{\tilde{\psi}}\right)^{1-u}=\left(x_{1}^{* q}+\omega y_{1}^{* q}\right)^{\frac{1}{q}}\left(\gamma_{2}^{* p}+\omega^{(1-p)} x_{2}^{* p}\right)^{\frac{1}{p}} \\
\leq x_{1} x_{2}+\gamma_{1} \gamma_{2}=\left\|f_{1}\right\|_{\psi}^{u}\left\|g_{1}\right\|_{\tilde{\psi}}^{1-u}+\left\|f_{2}\right\|_{\tilde{\psi}}^{u}\left\|g_{2}\right\|_{\tilde{\psi}}^{1-u}=\frac{\left\|f_{1}\right\|_{\psi}^{u}}{\left\|g_{1}\right\|_{\tilde{\psi}}^{u-1}}+\frac{\left\|f_{2}\right\|_{\psi}^{u}}{\left\|g_{2}\right\|_{\tilde{\psi}}^{u-1}} .
\end{gathered}
$$

Remark 24. Let $u \geq 1,1 \leq \omega$. Let $\psi \in \Psi, \tilde{\psi} \in \tilde{\Psi}$ and $0<\left\|g_{1}^{*}\right\|_{\tilde{\psi}} \leq \omega^{\frac{1}{u-1}}\left\|g_{2}^{*}\right\|_{\tilde{\psi}}$. Then

$$
\left\|f_{1}^{*}+\omega f_{2}^{*}\right\|_{\psi}^{u}\left(\left\|g_{1}\right\|_{\tilde{\psi}}^{1-u}+\left\|g_{2}\right\|_{\tilde{\psi}}^{1-u}\right)(\omega+1)^{-u} \leq \frac{\left\|f_{1}\right\|_{\psi}^{u}}{\left\|g_{1}\right\|_{\tilde{\psi}}^{u-1}}+\frac{\left\|f_{2}\right\|_{\psi}^{u}}{\left\|g_{2}\right\|_{\tilde{\psi}}^{u-1}} .
$$

We get this by replacing $x_{2}, y_{2}$ in the above corollary by $x_{2}=\left\|g_{1}\right\|_{\tilde{\psi}}^{1-u} y_{2}=\left\|g_{2}\right\|_{\tilde{\psi}}^{1-u}$. If we use the inequality $\left(\frac{a^{\alpha}+b^{\alpha}}{2}\right)^{\frac{1}{\alpha}} \leq \frac{a+b}{2}, \alpha=1-u \leq 0<1$ we get that

$$
\left(\left\|g_{1}\right\|_{\tilde{\psi}}^{1-u}+\left\|g_{2}\right\|_{\tilde{\psi}}^{1-u}\right)(\omega+1)^{-u} \geq\left(\left\|g_{1}\right\|_{\tilde{\psi}}+\left\|g_{2}\right\|_{\tilde{\psi}}\right)^{1-u} 2^{u}(\omega+1)^{-u} \geq\left\|g_{1}+g_{2}\right\|_{\tilde{\psi}}^{1-u} 2^{u}(\omega+1)^{-u} .
$$


Remark 25. For the case $\left\|g_{1}^{*}\right\|_{\tilde{\psi}} \leq \omega^{\frac{1}{u-1}}\left\|g_{2}^{*}\right\|_{\tilde{\psi}}$ we get another variant of the Beckenbach-Dresher inequality, namely

$$
\frac{\left\|f_{1}^{*}+\omega f_{2}^{*}\right\|_{\psi}^{u}}{\left\|g_{1}+g_{2}\right\|_{\tilde{\psi}}^{u-1}}\left(\frac{2}{\omega+1}\right)^{u} \leq \frac{\left\|f_{1}\right\|_{\psi}^{u}}{\left\|g_{1}\right\|_{\tilde{\psi}}^{u-1}}+\frac{\left\|f_{2}\right\|_{\psi}^{u}}{\left\|g_{2}\right\|_{\tilde{\psi}}^{u-1}} .
$$

Remark 26. The cases $u<0, \psi \in \tilde{\Psi}, \tilde{\psi} \in \Psi$ and $u<1, \psi \in \tilde{\Psi}, \tilde{\psi} \in \tilde{\Psi}$ can be treated in a similar way.

For the case $q \geq 1,0<\omega \leq 1,\|\cdot\|_{\omega, q}$ is a norm, so we have the Minkowski and the Hölder inequality (2).

For the case $q \leq 1, \omega \leq 1$ we have Minkowski inequality with constant $\frac{1}{2}(\omega+1)^{\frac{1}{q}}$, inverse Minkowski, and inverse Holder inequalities.

For the case $q \geq 1,1 \leq \omega$, we have Minkowski inequality with constant $((\omega+1) / 2)^{\frac{1}{q}}$. The function $\psi_{\omega, q}$ is not convex. It has relative minimums at the points $t_{0}$ and $t_{1}$. We could try to improve it constructing a convex function changing it on the interval $\left(t_{0}\right.$, $t_{1}$ ) by replacing it by a constant equal to $\psi_{\omega, q}\left(t_{0}\right)=\psi_{\omega, q}\left(t_{1}\right)$. The corresponding norm is a norm indeed, but some calculations show that actually this is not a new norm, but the norm $\|(x, y)\|_{\omega^{1-p, p}}^{*}=\|(x, y)\|_{\psi_{\omega^{1}-p, p}^{*}}$.

For the case $q \leq 1,0<\omega \leq 1$, we have the Minkowski inequality with constant $\frac{1}{2}(4 /(\omega+1))^{1 / q}$. The function $\psi_{\omega, q}$ is not concave. It has relative maximum at the points $t_{0}$ and $t_{1}$. We can improve it constructing a concave function, namely changing it on the interval $\left(t_{0}, t_{1}\right)$ by replacing it by a constant equal to $\psi_{\omega, q}\left(t_{0}\right)=\psi_{\omega, q}\left(t_{1}\right)$. The new function is

$$
\psi_{c, \omega, q}(t)= \begin{cases}\left((1-t)^{q}+\omega t^{q}\right)^{\frac{1}{q}}, & 0 \leq t \leq \frac{1}{1+\omega^{p-1}}, \\ \frac{\omega^{\frac{1}{q}}}{\left(1+\omega^{p-1}\right)^{\frac{1}{p}}}, & \frac{1}{1+\omega^{p-1}} \leq t \leq \frac{\omega^{p-1}}{1+\omega^{p-1}}, \\ \left(t^{q}+\omega(1-t)^{q}\right)^{\frac{1}{q}}, & \frac{\omega^{p-1}}{1+\omega^{p-1}} \leq t \leq 1,\end{cases}
$$

where $\frac{1}{p}+\frac{1}{q}=1$, and

$$
\|(x, y)\|_{c, \omega, q}= \begin{cases}\left(|x|^{\omega}+\omega|y|^{q}\right)^{\frac{1}{q}}, & \omega^{p-1}|x| \geq|y|, \\ \frac{\omega}{\left(1+\omega^{p-1}\right)^{q-1}}(|x|+|y|), & \omega^{1-1}|x| \leq|y| \leq \omega^{p-1}|x|, \\ \left(|y|^{q}+\omega|x|^{q}\right)^{\frac{1}{q}}, & \omega^{p-1}|x| \leq|y| .\end{cases}
$$

Remark 27. Analogous results connected to the function $\psi_{p, q, \lambda}$ are given in the article [22]. 
University SRF under contract no. 150/2011. The research of the third author was supported by the Ministry of Science, Education and Sports of the Republic of Croatia under grants 058-1170889-1050 and 037-1001677-2769.

\section{Author details}

'Department of Mathematics and Informatics, Sofia University, Sofia, Bulgaria ${ }^{2}$ Department of Mathematics, Luleå University of Technology, Luleå, Sweden ${ }^{3}$ Department of Mathematics, University of Zagreb, Zagreb, Croatia

\section{Authors' contributions}

All authors conceived of the study and carried out the proof. All authors read and approved the final manuscript.

\section{Competing interests}

The authors declare that they have no competing interests.

\section{Received: 14 July 2011 Accepted: 12 January 2012 Published: 12 January 2012}

\section{References}

1. Beckenbach, EF: A class of mean-value functions. Am Math Mon. 57, 1-6 (1950). doi:10.2307/2305163

2. Dresher, M: Moment spaces and inequalities. Duke Math J. 20, 261-271 (1953). doi:10.1215/S0012-7094-53-02026-2

3. Beckenbach, EF, Bellman, R: Inequalities. Springer, Berlin (1961)

4. Pečarić, JE, Beesack, PR: On Jessen's inequality for convex functions II. J Math Anal Appl. 118, 125-144 (1986). doi:10.1016/0022-247X(86)90296-9

5. Peetre, J, Persson, LE: A general Beckenbach's inequality with applications. In Function Spaces, Differential Operators and Nonlinear Analysis. Pitman Res Notes Math Ser. 211, 125-139 (1989)

6. Persson, LE: Generalizations of some classical inequalities with applications. Teubner Texte zur Mathematik. 119 127-148 (1991)

7. Varošanec, S: The generalized Beckenbach inequality and related results. Banach J Math Anal. 4(1):13-20 (2010)

8. Saito, KS, Kato, K, Takahashi, Y: Von Neumann-Jordan constant of absolute normalized norms on C $C^{2}$ J J Math Anal Appl. 244, 515-532 (2000). doi:10.1006/jmaa.2000.6727

9. Bonsall, FF, Duncan, J: Numerical Ranges II, London Math. In Soc Lecture Note Ser, vol. 10,Cambridge University Press, Cambridge (1973)

10. Mitani, Kl, Saito, KS: Dual of two-dimensional Lorentz sequence spaces. Nonlinear Anal. 71, 5238-5247 (2009). doi:10.1016/j.na.2009.04.007

11. Mitani, Kl, Oshiro, S, Saito, KS: Smoothness of $\Psi$-direct sums of Banach spaces. Math Inequal Appl. 8, 147-157 (2005)

12. Mitrinović, DS, Pečarić, JE, Fink, AM: Classical and New Inequalities in Analysis. Kluwer Acad. Publishers, Dordrecht (1993)

13. Kato, M, Saito, KS, Tamura, T: On $\psi$-direct sums of Banach spaces and convexity. J Aust Math Soc. 75, 413-422 (2003). doi:10.1017/S1446788700008193

14. Mitani, Kl, Saito, KS: A note on geometrical properties of Banach spaces using $\psi$-direct sums. J Math Anal Appl. 327 898-907 (2007). doi:10.1016/j.jmaa.2006.04.059

15. Saito, KS, Kato, M: Uniform convexity of $\psi$-direct sums of Banach spaces. J Math Anal Appl. 277, 1-11 (2003). doi:10.1016/S0022-247X(02)00282-2

16. Takahashi, Y, Kato, M, Saito, KS: Strict convexity of absolute norms on $\mathbf{C}^{2}$ and direct sums of Banach spaces. J Inequal Appl. 7, 179-186 (2002)

17. Dowling, PN: On convexity properties of $\psi$-direct sums of Banach spaces. J Math Anal Appl. 288, 540-543 (2003). doi:10.1016/j.jmaa.2003.09.011

18. Dhompongsa, S, Kaewkhao, A, Saejung, S: Uniform smoothness and U-convexity of $\psi$-direct sums. J Nonlinear Convex Anal. 6, 327-338 (2005)

19. Saito, KS, Kato, M, Takahashi, Y: Absolute norms on C ${ }^{n}$. J Math Anal Appl. 252, 879-905 (2000). doi:10.1006/ jmaa.2000.7139

20. Kato, M, Maligranda, L: On James and Jordan-von Neumann constants of Lorentz sequences spaces. J Math Anal Appl. 258, 457-465 (2001). doi:10.1006/jmaa.2000.7367

21. Kato, M: On Lorentz spaces I $(E)$. Hiroshima Math J. 6, 73-93 (1976)

22. Nikolova, L, Varošanec, $S$ : Refinements of Hölder's inequality derived from functions $\psi_{p, q, \lambda}$ and $\mathbb{\Xi}_{p, q, \lambda}$. Ann Funct Anal. 2, 72-83 (2011)

doi:10.1186/1029-242X-2012-7

Cite this article as: Nikolova et al:: The Beckenbach-Dresher inequality in the $\Psi$-direct sums of spaces and related results. Journal of Inequalities and Applications 2012 2012:7. 